\title{
Study on Calculation Method of Negative Pressure Loss Considering the Deformation of Drainage Borehole
}

\author{
Xue-Bo Zhang ${ }^{1,2,3}$, Jian-liang Gao ${ }^{1,2,3}$ \\ ${ }^{1}$ The Collaborative Innovation Center of Coal Safety Production of Henan Province \\ Jiaozuo, Henan, China 454003 \\ ${ }^{2}$ State Key Laboratory Cultivation Base for Gas Geology and Gas Control \\ Jiaozuo, Henan, China, 454003 \\ ${ }^{3}$ Henan Polytechnic University \\ Jiaozuo, Henan, China 454003 \\ zhxb@hpu.edu.cn; gao@hpu.edu.cn
}

\begin{abstract}
The experimental system for borehole negative pressure distribution and flow distribution was constructed. The laws of borehole negative pressure distribution and flow distribution under different negative pressure conditions with consideration of borehole deformation were obtained. The theory of calculating borehole negative pressure loss was analyzed. A negative pressure loss calculation method with consideration of borehole deformation was proposed based on experimental measured results, and its validity was verified comparing the results calculated by proposed method and experimental measured results. Results show that: 1) The negative pressure loss for complete hole is relatively small, the negative pressures at different parts of borehole are similar with each other, and the borehole extraction flow is approximately in a linear distribution along borehole length direction. The larger the orifice pressure is, the larger the borehole extraction flow is, and the larger the borehole negative pressure loss is (it is worth noting that borehole negative pressure is still much smaller than orifice pressure). 2) In the case of hole-bottom collapse, the negative pressure loss in the collapse section is larger than that in complete section. Since the flow in the collapse section is small, its negative pressure loss is small as well. The total negative pressure loss upon hole collapse is larger than that in complete hole, while its total extraction flow is slightly smaller than that of complete hole. The hole-bottom collapse does not significantly affect the gas extraction performance. 3) The negative pressure loss results, which is calculated by the proposed method based on the consideration of borehole deformation, are consistent with the experiment results. Therefore, the proposed method can be used to calculate the negative pressure loss of extraction borehole.
\end{abstract}

Keywords: Borehole deformation, Drainage borehole, Negative pressure loss, Resistance coefficient.

\section{Introduction}

Extraction negative pressure is a key parameter in borehole gas extraction. The negative pressure loss calculation method considering borehole deformation can be used to obtain negative pressure distribution under different borehole deformation conditions, and provides theoretical basis for analyzing the influence of borehole deformation to gas extraction as well as the development of technologies against borehole deformation instability. Numerous domestic scholars have made researches on borehole negative loss. Xin Xinping [1] measured the negative pressure loss in coal seam gas extraction and found that the negative pressure loss was insignificant when the extraction time was short, while with the extending of extraction time, the borehole wall deformation would enlarge the negative pressure loss along borehole. Li Wei et al. [2] concluded that the extraction borehole negative pressure loss in loaded coal seam was relatively smaller, while that in unloaded coal seam was relatively larger. Ji Zhongchao, Li Shuwen $[3,4]$ both calculated borehole negative pressure loss using hydromechanical Frictional resistance formula, and believed that the borehole negative pressure loss was smaller than the orifice pressure loss. $\mathrm{Hu}$ Peng [5] made experiment and found that the negative pressure distribution along borehole length followed a logarithmic curve distribution. Li Jie [6] carried out site test and believed that borehole negative pressure was in a linear distribution along the borehole length direction. Liu Jun [7] conducted experiment can obtained the function relationship among borehole negative pressure, borehole length and flow, and applied such function relationship in numerical analysis. There are many domestic and foreign scholars conducting large amounts of researches on the horizontal well pressure drop in oil exploitation [8-13], and great achievements have been obtained. Su et al.[13] divided the pressure drop of horizontal well into frictional 
pressure drop, acceleration pressure drop, mixing pressure drop, and presented calculation formulas for frictional pressure drop and acceleration pressure drop using hydrodynamic theory. The mixing pressure drop could not be obtained by generalized formula but can only obtain by experimental measurement.

Based on above studies, it can be known that current calculation methods of negative pressure loss are far from perfect; especially the negative pressure calculation method considering borehole deformation needs to be further improved. The study of the calculation method of borehole negative pressure should take variable mass flow of gas migration in borehole into consideration. The borehole negative pressure loss should be comprehensively analyzed based on borehole extraction negative pressure distribution, borehole flow distribution and borehole deformation. In this paper, a negative pressure loss calculation method considering borehole deformation was proposed according to the variable mass flow of gas migration in borehole through theoretical analysis and experimental measurement.

\section{Theoretical Analysis of the Calculation of Negative Pressure Loss in Gas Extraction Borehole}

During the flowing process of gas in borehole, gas continuously discharge from hole wall, and the gas mass in borehole varies constantly, which is called variable mass flow. As shown in Fig.1, due the friction between gas and hole wall, variation of gas flow, mixing of radial flowing gas and horizontal flowing gas, and borehole deformation, extraction negative pressure losses including loss due to frictional resistance, acceleration loss, mixed loss and local loss will occur.

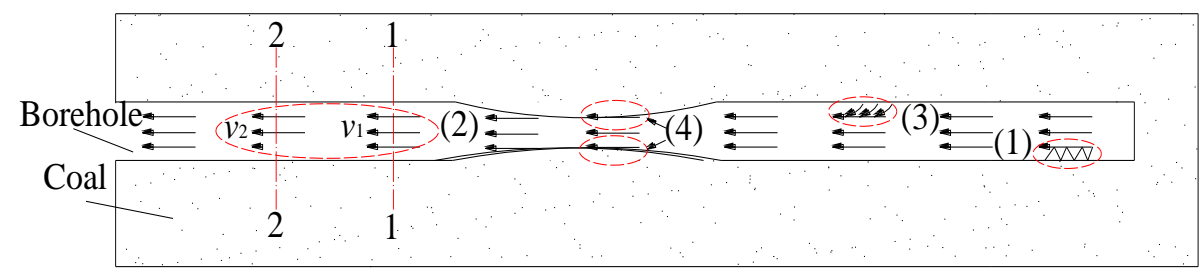

(1) loss due to frictional resistance (2) acceleration loss (3) mixed loss (4) local loss

Fig. 1: Classification of negative pressure loss in boreholes.

Loss due to frictional resistance refers to the resistance loss caused by friction between gas and borehole wall as well as the disturbance and friction between gas molecules. From the borehole bottom to orifice, the gas flow through the cross section becomes larger and larger; moreover, the more it is close to the cross section, the larger the flow velocity is. When the shrinkage cavity of borehole is observed, the cross-section velocity close to the orifice will be even larger. The radial flowing gas has certain velocity, which is perpendicular to the velocity direction of axial flowing gas. Mix loss will occur when they meet each other. The larger the permeability coefficient in coal seam is, the larger the discharge amount of gas from borehole wall is, and thus the larger the mixed loss is. Currently, mixed loss cannot be expressed by a certain formula, but can only obtain by approximation of experimental results. In this study, the mixed loss was obtained by correcting resistance coefficient based on experimental results of negative pressure loss. The borehole deformation or instability will cause the change of borehole diameter. Sudden increase or decrease of borehole diameter will lead to certain pressure loss, i.e. local loss. The local loss can be calculated using local resistance coefficient method, wherein the local loss is expressed as a function of kinetic energy factor.

The negative pressure loss in extraction borehole is equal to the sum of loss due to frictional resistance, acceleration loss, mixed loss and local loss:

$$
\Delta p=\Delta p_{\text {wall }}+\Delta p_{\text {acc }}+\Delta p_{\text {mix }}+\Delta p_{f}=f \frac{\Delta L}{d} \frac{\rho \bar{v}^{2}}{2}+\rho\left(v_{1}{ }^{2}-v_{2}{ }^{2}\right)+\Delta p_{\text {mix }}+\xi \frac{\rho v^{2}}{2}
$$

Where $\Delta p_{\text {wall }}$ is loss due to frictional resistance, $\mathrm{Pa} ; d$ is borehole diameter, $\mathrm{m} ; \rho$ is gas density in borehole, $\mathrm{kg} / \mathrm{m}^{3} ; f$ is resistance coefficient; $\bar{v}$ is average wind velocity in borehole, $\mathrm{m} / \mathrm{s} ; \Delta p_{a c c}$ is acceleration loss, $\mathrm{Pa} ; v_{1} 、 v_{2}$ _ gas velocity at both ends of borehole, $\mathrm{m} / \mathrm{s} ; \Delta p_{f}$ is local loss, $\mathrm{Pa} ; v$ is gas velocity, $\mathrm{m} / \mathrm{s}$, which is normally regarded as the gas velocity 
after local loss; $\xi$ is local resistance coefficient, which can be calculated by local resistance coefficient method corresponding to underworkings.

After revising mixed loss using resistance coefficient, the equation above can be converted into:

$$
\Delta p=f_{i} \frac{\Delta L}{d} \frac{\rho \bar{v}^{2}}{2}+\rho\left(v_{1}^{2}-v_{2}^{2}\right)+\xi \frac{\rho v^{2}}{2}
$$

Equation 2 is the calculation equation of borehole negative pressure loss considering borehole deformation, where $f_{i}$ is the actual resistance coefficient with consideration of mixed loss. To obtain the calculation method of $f_{i}$, experiments on the borehole negative pressure distribution and flow distribution under the conditions of borehole deformation and instability were conducted.

\section{Experiment on Negative Pressure Distribution and Flow Distribution under Different Degrees of Borehole Deformation and Instability}

\subsection{Design of Test System of Borehole Negative Pressure Distribution}

Suppose the permeability of coal seam is a constant value, the gas flowing velocity is only correlated to the gas pressure gradient, and the gas flow field in coal seam is basically uniform. The designed test system of borehole negative pressure distribution consists of air extracting system, pressure adjusting system, pipeline system, and pressure/flow test system.

1) Air extracting system: air extracting system consists of water ring pump, gas-water separator, water intake apparatus, and water-freeing arrangement.

2) Pressure adjusting system: the negative pressure of pipeline system can be adjusted by rotating the pressure-adjusting valve at the outlet of vacuum pump.

3) Pipeline system: as shown in Fig.2, the pipeline system consists of filling box, filler and borehole. Considering that in actual situation the gas always flows into borehole, the borehole is fabricated in a cylindrical structure made of rolled fine wire, locating in the middle of pipeline system. Sieve pores are made as air inlets on packing box. By filling the same weight of materials into the pipeline of the same length, the gas permeability of pipelines can be guaranteed to be identical. Then, the sufficient amount of sieve pores in uniform distribution should be made on packing box, in order to guarantee a uniform flow field in pipeline system.

4) Test system of pressure (flow): arrange pressure (flow) measure points in pipeline at regular distance intervals, then the pressure, flow rate and flow quantity can be measured using pitot tube and U-shape mercury meter.

This experimental system adopts air as medium, and the pressure in/around filler is set to be one atmosphere. When the system is operating, the air will be continuously sucked into filler under the effect of pressure difference (caused by extraction negative pressure) and then flow into borehole. The designed test system of extraction negative pressure distribution is $52 \mathrm{~m}$ in length, including borehole length of $50 \mathrm{~m}$ (measured section is $46.9 \mathrm{~m}$ ), the borehole diameter is $0.1 \mathrm{~m}$, fine sands are filled around borehole and then pressed tight into a square structure with dimension of $0.4 \mathrm{~m} \times 0.4 \mathrm{~m}$.

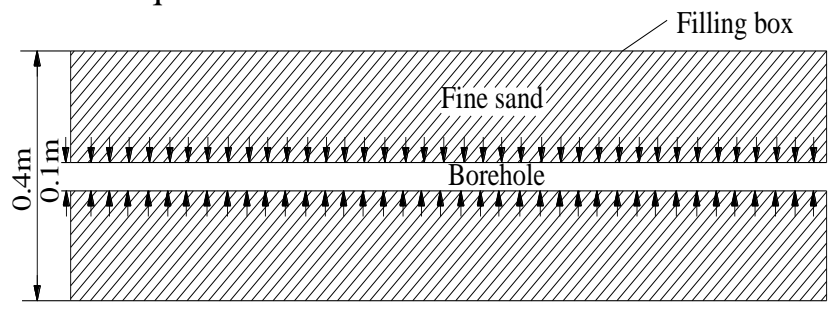

Fig. 2: Schematic diagram of piping system profile

\subsection{Experiment Scheme}

Experiments of borehole negative pressure distribution and flow distribution for complete hole and collapsed hole were conducted, respectively.

1) Measurement of negative pressure distribution and flow distribution in complete borehole. The borehole diameter is maintained to be $0.1 \mathrm{~m}$, without deformation. Then the static pressure and dynamic pressure at all measure points under 
different extraction negative pressures were measured, so that the negative pressure distribution and flow distribution of the system can be obtained.

2) Measurement of negative pressure distribution and flow distribution in collapsed borehole. Due to the influence of in situ stress, the collapse degree and collapse site are varying. In this study, the borehole section which locates $0 \sim 18.8 \mathrm{~m}$ away from borehole bottom was collapsed, in which the borehole diameter was shrunk by 50\%, while the other sections remained complete. After the borehole diameter within collapsed section was shrunk to $0.05 \mathrm{~m}$, the same amount of fine sand were filled in, and the gas permeability of sand structure around borehole was increased (in contrast, the gas permeability of complete section of borehole remained unchanged). The negative pressure distribution and flow distribution under different extraction negative pressures were measured.

\subsection{Analysis of Experimental Results}

1) The law of extraction negative pressure distribution and flow distribution of complete borehole

When the borehole cross-section is complete without deformation, the distributions of negative pressure and flow along the length direction are shown as Fig.3 and 4, respectively.

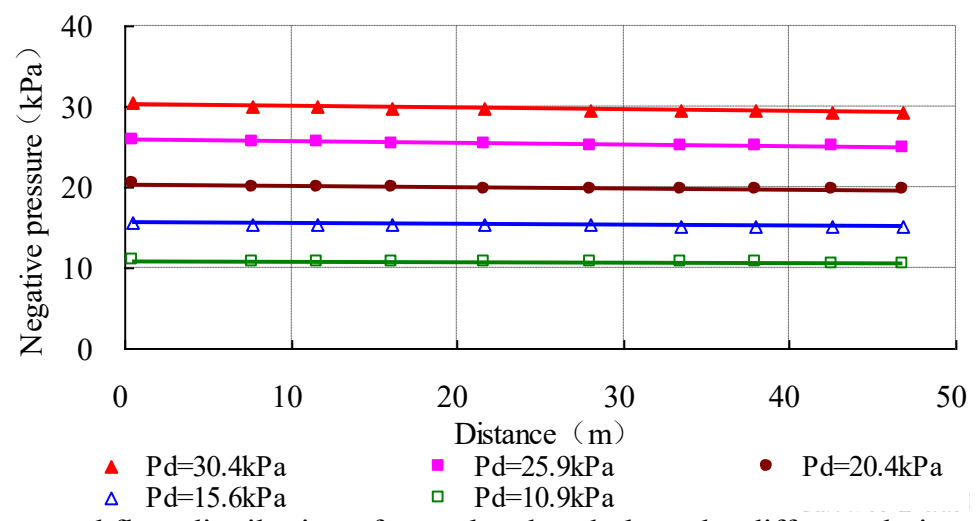

Fig. 3: Negative pressure and flow distribution of complete borehole under different drainage negative pressure.

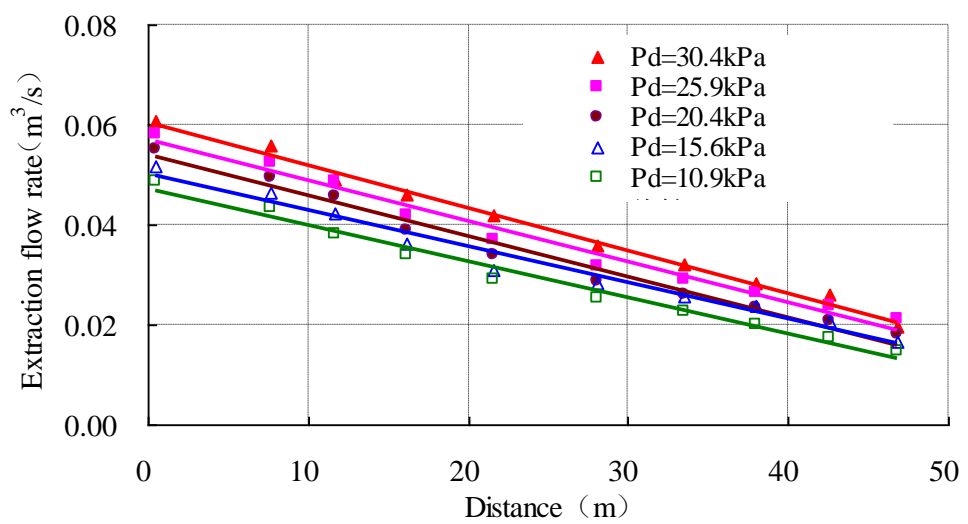

Fig. 4: Flow distributions in the complete borehole under different drainage negative pressure.

As shown in Fig.3 and 4, the negative pressure loss of complete borehole is relatively smaller, the negative pressures at different sites are similar with each other, and the gas extraction amount at different sites are similar as well, therefore it can be known the gas extraction flow is approximately in linear distribution along length direction. The larger the negative pressure at orifice is, the larger the extraction flow is, and the larger the negative pressure loss is (of course, it is still far smaller than negative pressure at orifice. As the negative pressure at orifice increases from $10.9 \mathrm{kPa}$ to $30.4 \mathrm{kPa}$, the total extraction flow increases from $0.049 \mathrm{~m} 3 / \mathrm{s}$ to $0.061 \mathrm{~m}^{3} / \mathrm{s}$, the negative pressure loss at bottom increases from $0.32 \mathrm{kPa}$ to 1.10 $\mathrm{kPa}$, accounting for only $2.93 \%-3.62 \%$ of negative pressure at orifice.

\section{2) The law of extraction negative pressure distribution and flow distribution of collapsed borehole}


When the borehole bottom is collapsed, the borehole diameter decreases from $0.1 \mathrm{~m}$ to $0.05 \mathrm{~m}$, and the distributions of negative pressure and flow along the length direction are shown as Fig.5 and 6, respectively.

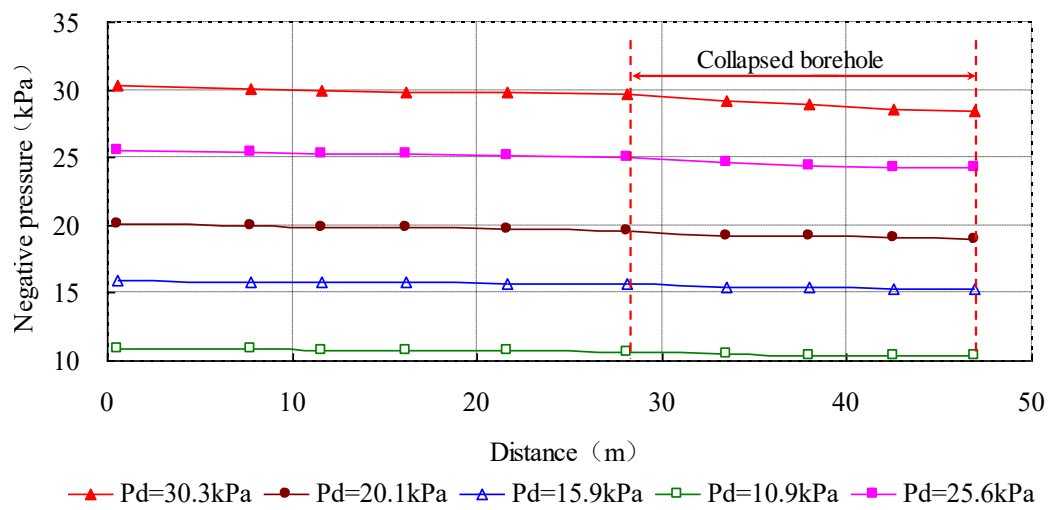

Fig. 5: Negative pressure distribution of borehole collapse under different drainage pressure.

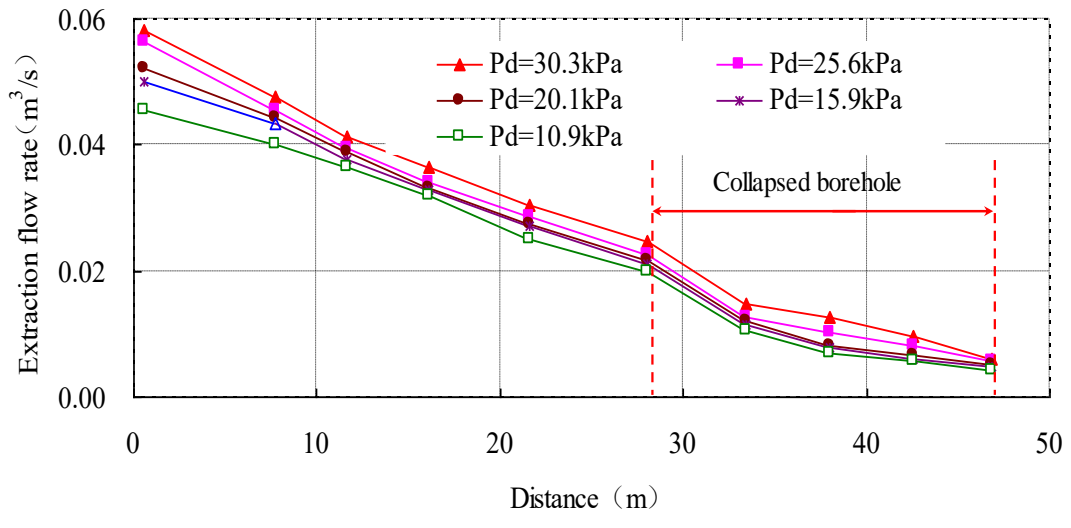

Fig. 6: Flow distribution of borehole collapse under different drainage pressure.

As shown in Fig.5 and 6, the negative pressure loss of collapsed section is relatively larger than that of complete section. As the collapse took place at the bottom, the borehole cross-section of collapsed section is decreased, which makes the flow quantity in borehole even smaller, so the negative pressure loss is not significant. The larger the extraction negative pressure is, the larger the extraction flow quantity is, and the larger the total negative pressure loss is. As the negative pressure increases from $10.9 \mathrm{kPa}$ to $30.3 \mathrm{kPa}$, the total extraction flow increases from $0.51 \mathrm{kPa}(0.30 \mathrm{kPa}$ at collapsed section) to $1.86 \mathrm{kPa}$ (1.22 kPa at collapsed section), accounting for only $4.68 \%-6.14 \%$ of negative pressure at orifice. Compared with complete hole, the total negative pressure loss of collapsed hole is relatively larger, while its total extraction flow quantity is slightly smaller. Therefore, it can be known that the bottom collapse does not significantly affect gas extraction performance.

\section{Presentation and Verification of Calculation Method of Negative Pressure Loss Considering Borehole Deformation}

\subsection{Presentation of Calculation Method of Negative Pressure Loss Considering Borehole Deformation}

As shown in Fig.7, when calculating borehole negative pressure loss, the borehole is first disserted into several differentiation elements along the length direction, and then each differentiation element is calculated. Regarding the $d x$ infinitesimal section at site $x$, the total negative pressure loss of $d x$ infinitesimal section can be obtained by equation of continuity and calculation model of borehole negative pressure loss.

$$
\frac{d p(x)}{d x}=\Delta p_{f i r}+\Delta p_{a c c}=\frac{2 f_{i} \rho[2 Q(x)+q(x)]^{2}}{\pi^{2} d^{5}}+\frac{16 \rho\left[q^{2}(x)+2 Q(x) q(x)\right]^{2}}{\pi^{2} d^{4}}+\frac{16 \zeta \rho[2 Q(x)+q(x)]^{2}}{\pi^{2} d^{4}}
$$


Where $p(x)$ is the borehole extraction pressure at site $x, \mathrm{~Pa} ; d^{\prime}$ is equivalent borehole diameter after deformation, $\mathrm{m}$; $Q(x)$ is gas extraction amount at site $x, \mathrm{~m}^{3} / \mathrm{min}$.

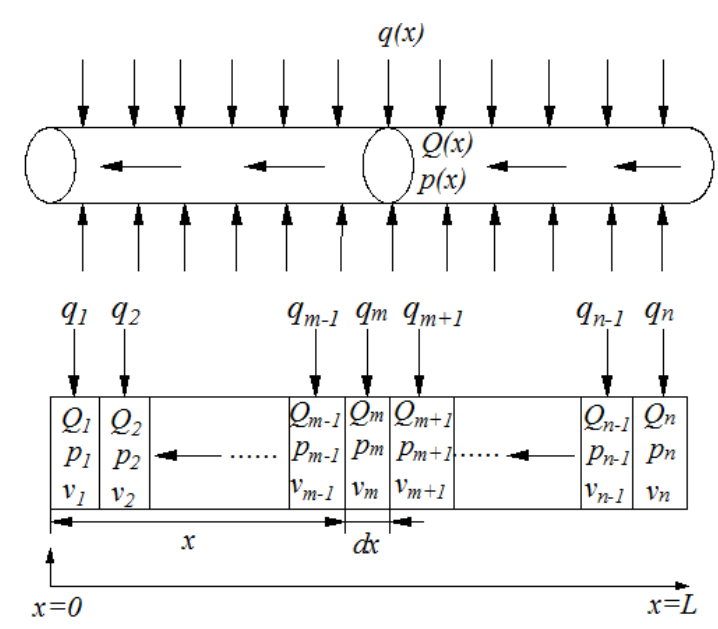

Fig. 7: Schematic diagram of bedding borehole separation along the direction of borehole.

In equation 3 , the resistance coefficient for borehole wall correction is $f_{i}$, which can be calculated according to the gas flow state of infinitesimal section. Concrete calculation processes are shown as follow:

1) Judging the gas flow state of infinitesimal section. First, the Reynolds number (Re) of each infinitesimal section was calculated by the dimension and flow rate of infinitesimal section, then the gas flow status can be judged.

2) Calculating resistance coefficient when there is no gas discharging from borehole wall. Many scholars have studied the resistance coefficients of fluids in different flow state on the basis of Nikuradse experimental results [14-16], and proposed corresponding calculation method for each resistance coefficient. According to the obtained gas flow state of each infinitesimal section, the resistance coefficient when there is no fluid flowing into borehole can be calculated using proper equation.

The resistance coefficient of fluid in Laminar flow state was calculated using the formula deduced from Hagen-poiseuille theory, and the result is consistent with Nikuradse experiment result, i.e.

$$
f_{0}=64 / \operatorname{Re} \quad(\operatorname{Re} \leq 2320)
$$

Where Re is Reynolds number, $\operatorname{Re}=v d / v$, $\mathrm{v}$ is the average flow velocity in cross-section, $v$ is coefficient of kinematic viscosity.

Due to the complexity of turbulent flow, it is very difficult to calculate the resistance coefficient with an exact theoretical formula. The resistance coefficient of fluid in turbulent flow state was calculated by Jain's semi-rational formula which is suitable for hydraulic smooth zone and hydraulic roughness region [16]:

$$
f_{0}=\left[1.14-2 \log \left(\varepsilon / d+21.25 \operatorname{Re}^{-0.9}\right)\right]^{-2} \quad(\operatorname{Re} \geq 4000)
$$

Where $\varepsilon$ is tube wall roughness, $\mathrm{m}$.

3) Calculation of actual borehole resistance coefficient. In actual borehole, gas continuously flows from wall into borehole. The gas flow in borehole belongs to a variable mass flow, of which the resistance coefficient cannot be calculated by above equation 4,5 any longer. In this study, the equation 4 and 5 were modified based on the experimental results of borehole negative pressure distribution and flow distribution as well as the results of previous researches, then the actual borehole resistance coefficient fi was calculated by such modified equation with consideration of mixed loss. On the basis of experimental results of borehole negative pressure distribution and the semi-rational formula (proposed by Ouyang et al.) of resistance coefficient fi when there is fluid flowing from hole wall into borehole, the calculation equation for actual borehole resistance coefficient fi with consideration of mixed loss was proposed. 


$$
\begin{gathered}
f_{i}=64 / \operatorname{Re} \times\left(1+0.04304 \operatorname{Re}^{0.6142}\right) \quad(\operatorname{Re} \leq 2320) \\
f_{i}=\left[1.14-2 \log \left(\varepsilon / d+21.25 \operatorname{Re}^{-0.9}\right)\right]^{-2} \times\left(1+0.0163 \operatorname{Re}^{0.2278}\right) \quad(\operatorname{Re} \geq 4000)
\end{gathered}
$$

The frictional resistance coefficient of fluid within transition section can be obtained by linear interpolation between above two equations.

\subsection{Validation of Calculation Method of Negative Pressure Loss Considering Borehole Deformation}

According to the calculation equation of borehole negative pressure loss considering mixed loss and based on the test condition of negative pressure distribution and measured flow distribution, the negative pressure loss for complete and collapsed hole was calculated, respectively. The calculation results were compared with experimental measured results, as shown in Fig.9 and 10.

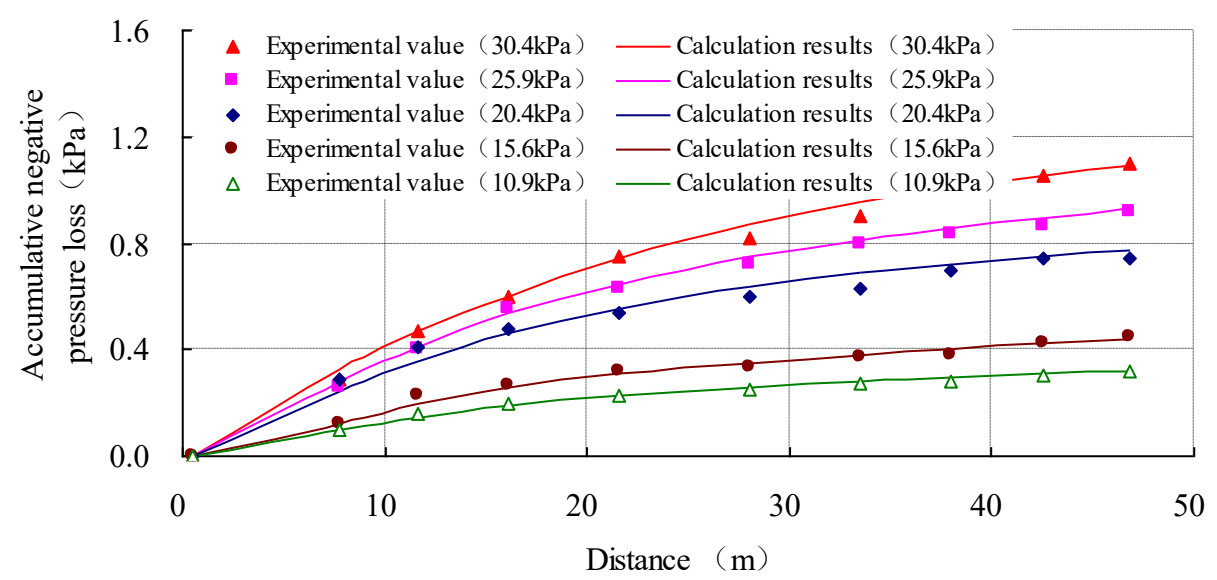

Fig. 8: Comparison of calculated results of negative pressure loss with experimental results (complete borehole).

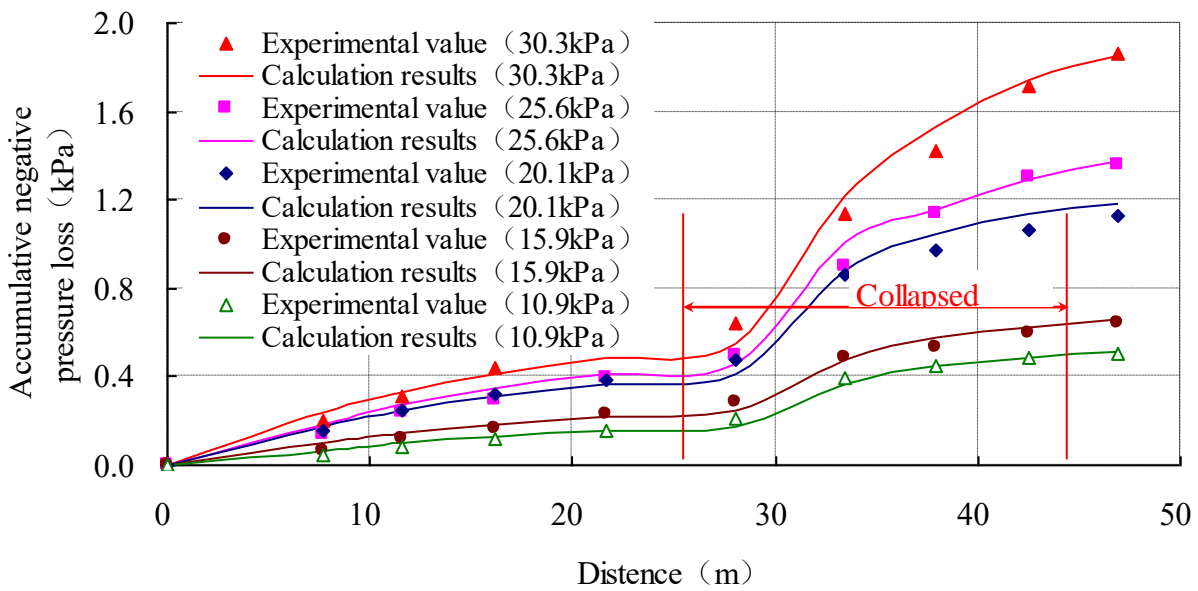

Fig. 9: Comparison of calculated results of negative pressure loss with experimental results (borehole collapse).

As shown in Fig.8 and 9, the negative pressure loss calculated by equation considering borehole deformation is consistent with the experimental results in terms both distribution law and value, therefore the calculation equation considering borehole deformation is suitable for calculating borehole negative pressure.

\section{Conclusions}


1) The experiment system of negative pressure distribution and flow distribution in gas extraction was constructed, and the negative pressure distribution and flow distribution under borehole deformation and stability were measured. Results showed that the negative pressure loss of complete hole was relatively smaller, the negative pressures at different sites were similar to each other, and the extraction flow was in a approximately linear distribution along length direction. The larger the negative pressure at orifice is, the larger the gas extraction amount is, and the larger the borehole negative pressure loss is (of course it is still far smaller than the negative pressure at orifice).

2) When the hole bottom was collapsed, the negative pressure loss of collapsed hole section was larger than that of complete hole section. Since the flow quantity in collapsed section was small, the negative pressure loss was not significantly. Compared with complete hole, the collapsed hole had a larger total negative pressure loss but a smaller total gas extraction amount. Therefore, it can be known the bottom collapse does not significantly affect the gas extraction performance.

3 ) The calculation theory of borehole negative pressure loss was analyzed. Based on the experimental results of negative pressure distribution and flow distribution under borehole deformations and instabilities, a calculation method of borehole negative pressure loss considering borehole deformation was proposed, and the validity of proposed method was verified. The negative pressure results calculated by the method considering borehole deformation are consistent with experimental results. Therefore, the calculation method considering borehole deformation can be used to calculate negative pressure loss.

\section{Acknowledgements}

Supported by the National Natural Science Foundation of China (Grant No. 51734007, No.51704099), Henan Province Basic and Frontier Technology Research Projects of China (Grant No.142300413233), Henan Province Major Scientific and Technological Breakthrough Research Project (Grant No.13B440038) and Opening Foundation of State Key Laboratory Cultivation Base for Gas Geology and Gas Control (Grant No.WS2017B14).

\section{References}

[1] X.-P. Xin, "Optimization of gas drainage parameters of coal seam in Jiaozuo mining area," Safety in Coal Mines, no. 9, pp. 7-10, 1998.

[2] W. Li, J.-X. Chen, “Analysis of gas drainage parameters in Luling Mine,” China Coal, vol. 26, no. 8 pp. 22-26, 2000.

[3] Z.-C. Ji, "Numerical simulation of gas drainage radius in borehole," M.S. Thesis, Dept. Safety Engineering. Henan Polytechnic University, 2012.

[4] S.-W. Li, "Study on negative pressure distribution in different depth of borehole in Xin'an mine," Coal engineering, no. 5 pp. 103-105, 2013.

[5] P. Hu, "Study on the distribution of vacuum in a gas drainage borehole and coal seam," M.S. Thesis, Dept. Safety Engineering. Henan Polytechnic University, 2009.

[6] J. Li, "Research on extraction effect change law along the drainage drilling hole," M.S. Thesis, Dept. Safety Engineering. Henan Polytechnic University, 2012.

[7] J. Liu, "Study on dynamic character and the influence of negative pressure along axial direction of gas drainage bore,’.Ph.D. dissertation, Dept. Safety Engineering. Henan Polytechnic University, 2014.

[8] B. J. Dikken, "Pressure drop in horizontal wells and its effect on their production performance," Journal of Petroleum Technology, vol. 42, no. 11 pp. 1426-1433, 1990.

[9] A. D. Hill, D. Zhu, "The relative importance of wellbore pressure drop and formation damage in horizontal well," Spe Production \& Operations, vol. 23, no. 2, pp. 232-240, 2008.

[10] P. Le, X.-F. Chen, Y. Fu, "A new computation model for pressure drop of variable mass flow in horizontal wells," Acta Petrolei Sinica, vol. 35, no. 1, pp. 93-98, 2014.

[11] Z.-M. Wang, T.-F. Zhao, L. Xu, "Study on the law of variable mass turbulent pressure drop of horizontal wellbore in perforated completion," Journal of the University of Petroleum, vol. 27, no. 1, pp. 41-44, 2003.

[12] X.-P. Liu, Z.-S. Zhang, X.-E. Liu, "Calculation model of flow pressure drop coupled with seepage in horizontal wellbore," Journal of Southwest Petroleum Institute, vol. 22, no. 2, pp. 36-39, 2000.

[13] Z. Su, J. S. Gudmundsson, "Perforation inflow reduces frictional pressure loss in horizontal wellbores," Journal of Petroleum Science and Engineering, no. 19, pp. 223-232, 1998.

[14] F.-T. Li, "A summary of the existing formula of pipe resistance coefficient," Progress in science and technology of water conservancy and hydropower, no. 03, pp. 53-61, 1986. 
[15] A. K. Jain, "Accurate explicit equation for Friction Factor," Journal of the Hydraulics Division, vol. 102, no. 5, pp. 674-677, 1976.

[16] P. K, Swamee, A. K. Jain, "Explicit eqations for pipe-flow problems," Journal of the Hydrauli Division, vol. 102 , no. 5, pp. 567-664, 1976. 